\title{
Experience of Emergency Cervical Tracheal Repair after Non-Iatrogenic Injury
}

\author{
Yasser Shaban Mubarak, MD, ${ }^{1}$ Mohamed Abdel Bary, $\mathrm{MD}^{2}$ \\ ${ }^{1}$ Minia University, Egypt; ${ }^{2}$ South Valley University, Egypt
}

\section{ABSTRACT}

Background: Non-iatrogenic cervical tracheal injury is very rare and challenging for emergency critical care, early diagnosis, and immediate repair. So, emergency repair after early definitive diagnosis is the mainstay to avoid mortality and morbidity.

Methods: A retrospective, observational, cohort study is undertaken with collected data on 50 patients with emergency cervical tracheal repair after non-iatrogenic injury between January 2011 to January 2020 at our accident and emergency department in Minia and South Valley Universities.

Results: The non-iatrogenic injury of the cervical trachea is more common in adult males (98\%), and blunt trauma $(70 \%)$ is the predominant type of injury. Subcutaneous emphysema (SE) is the most common presentation (99\%). There are associated comorbidities like vascular (4\%), maxillofacial (6\%), head injury (2\%), and esophageal tear (8\%). Successful intubation is lifesaving, and early diagnosis and repair avoid complications.

Conclusions: Early diagnosis is a cornerstone for the successful management of cervical tracheal injury, in which successful intubation is a mainstay to reduce mortality. Emergency surgical repair reduces mortality and complications.

\section{INTRODUCTION}

While cervical tracheal injuries infrequently occur, it has been reported that about $75 \%$ of victims immediately died due to respiratory failure, airway obstruction, or tension pneumothorax or head injury [Zhao 2017; Eduardo 2009; Weil 2019]. Also, late complications were reported, such as tracheal stenosis or repeated chest infections. Most of cervical tracheal injuries occur due to blunt, penetrating, or iatrogenic cervicothoracic trauma [Yasser 2018]. The cervical trachea was injured in $6 \%$ of cases with penetrating tracheobronchial injury (TBI), and it was associated with esophageal injuries in $43 \%$ of the cases. However, it was affected in $0.4 \%$ of blunt TBI cases [Yasser 2018; Prokakis 2014]. Endotracheal tube (ETT) may be necessary, if catastrophic events result in tracheal injury. ETT under laryngoscopy guidance is the key point to gain airway patency, good ventilation, and avoid worsening injury [Cheng-Yu 2011]. Bronchoscopy and chest

Received September 23, 2020; accepted November 2, 2020

Correspondence: Yasser Shaban Mohamed Mubarak, Minia University, Telephone 01002554078 (e-mail: yassermubarak73@gmail.com) computed tomography (CT) with $M P R / 3 D$ reconstruction of the airway represent the procedures of choice for definitive diagnosis [Beshay 2020]. Accordingly, cervical tracheal injuries present a unique challenge to the anesthesiologists and surgeons. So, the early detection and emergency airway resumption are the cornerstones for survival [Yasser 2018].

To our knowledge, no large reports about the management of non-iatrogenic cervical tracheal injuries have been studied. We aim to present our clinical and surgical experience for the management of emergency non-iatrogenic cervical tracheal injuries with emphasis on natural history and early and late postoperative outcomes.

\section{METHODS}

Study design: This is a retrospective study that included 50 patients with non-iatrogenic cervical tracheal trauma. They were presented to Minia University Hospital between January 2011 to January 2020. The study conforms to the ethical standards of the Helsinki Declaration and approval was obtained from the IRB of Qena Faculty of Medicine and FMREC of Minia Faculty of Medicine. The data of all patients were collected from hospital records and personal patients contact.

Surgical management: In the emergency department, airway control was done for all cases by aspiration of secretions and maintenance of oxygenation. ETT was inserted with assisted laryngoscopy in cases of patients' desaturation, severe air leak from the neck wound, unstable general condition, or loss of consciousness. However, tracheostomy was performed in cases with severe maxillofacial injuries or failed ETT insertion. A high index of suspicion in all cases and diagnosis was confirmed by bronchoscopy (rigid or fiberoptic), and MDCT neck or VB, according to the stability of the general condition. (Figure 1)

The decision-making was done by a team of anesthetists, neurosurgeons, otolaryngologists, general, vascular, and thoracic surgeons. A low collar incision $2 \mathrm{~cm}$ above the sternum or extended neck wound for exploration and repair was done, while the neck is hyperextended provided there was no cervical spine fracture. If needed, the orotracheal tube was withdrawn and replaced by another sterile tube through the distal trachea in the operative field and returned oral ETT after repair in cases of complete tracheal transection. Extensive dissection of the trachea was avoided for neurovascular protection. Tracheal injury was repaired using $3 / 0$ vicyrl interrupted sutures with knots outside, starting from the posterior membranous and finishing with anterior cartilaginous. The esophageal injury detected during exploration should be repaired at the same time by $3 / 0$ silk interrupted sutured in two layers, including 

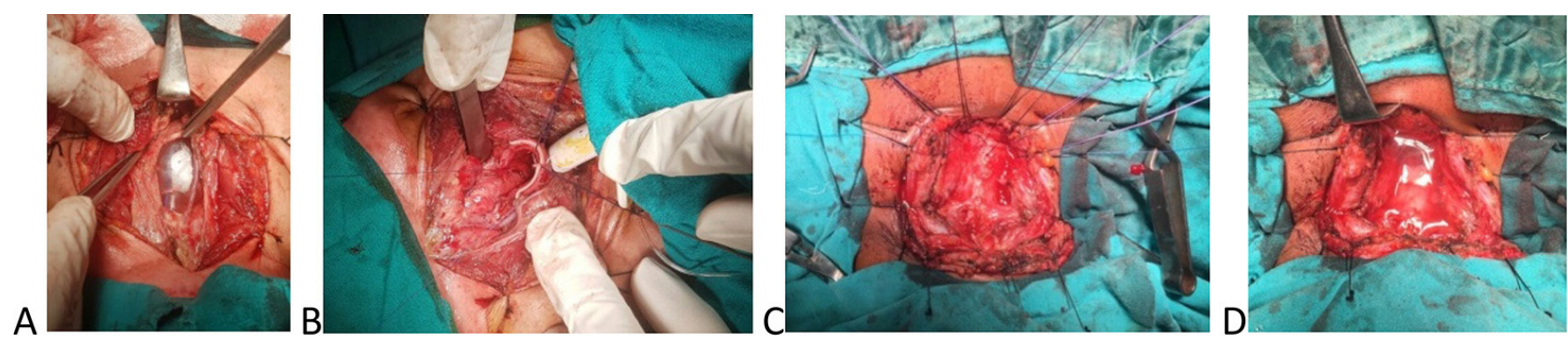

Figure 1. (A) Cervical tracheal injury with ETT balloon inside; (B) Complete tracheal transection; (C) Complete tracheal repair; (D) Saline test for air leak

the mucosa, musculosa, and serosa. A nasogastric tube (NGT) previously was inserted and left for two weeks. After completion of tracheal and esophageal repair, muscle flap was interposed between both to avoid fistula formation and give vascularity to anastomotic sites. Testing of anastomotic site leak was done by saline during deflated ETT cuff ventilation. The incision was anatomically closed in layers with a tubal drain away from the anastomotic sites. A stay suture from the chin to the sternum was done for a week. The mobility of the vocal cords was visualized by laryngoscopy after extubation. Collected data were done about mortality, vocal cord paralysis, esophageal leakage, and tracheal anastomotic site dehiscence.

Statistical analysis: The data were presented as mean, standard deviation, range, or percentage.

\section{RESULTS}

Preoperative and intraoperative data: Fifty patients presented with non-iatrogenic cervical tracheal trauma, 49 (98\%) of them were males, and their age range was $20-40$ years $(30 \pm 10$ years). Blunt trauma (MCA, falls from height, and clothesline) was the cause in $35(70 \%)$ cases and penetrating trauma (stab, firearm, or glass) in $15(30 \%)$ cases.

Subcutaneous emphysema was observed in 49 (99\%) of cases, hemoptysis in $15(30 \%)$, neck contusions in $15(30 \%)$, one case (2\%) with respiratory distress, and one case ( $2 \%)$ with open neck wound and air leak. The incidence of associated injuries was four (8\%) cases with esophageal perforation, four $(8 \%)$ cases with vascular injury, one $(2 \%)$ case with head injury that needed surgery, and three (6\%) cases with maxillofacial injuries. There was easy intubation in $46(92 \%)$ cases, tracheostomy was performed in three $(6 \%)$ cases, and insertion of ETT into the distal end of the transected trachea through the wound was done one case (2\%). The tracheal injury successfully was repaired in all cases. Esophageal repair was done in three cases with insertion of NGT. Vascular surgery intervention was required in two cases, ligation of the external jugular vein (EJV) in one case and ligation of the internal jugular vein in the other case. Severe concomitant injuries were reported in two cases $(4 \%)$, one of them was associated with esophageal injury, severe brain damage, and extradural hematoma that required neurosurgical intervention. The other case was associated with severe vascular, esophageal, and maxillofacial injuries. The hemorrhage was profuse, due to injury of IJV and was very difficult to control after removal of local compression. Emergency exploration was done with proximal and distal vascular control by ligation of both ends with $3 / 0$ polypropylene. The risk of embolization to heart and pulmonary embolism, cerebral and face congestion was a disastrous consequence. All cases were extubated on the table except for the two cases that were associated with severe concomitant injuries; they were intubated and transferred to ICU.

Postoperative data: The postoperative course was uneventful in other cases, and the patients were discharged on average after 9.5 days (range, 7-14 days). The 30-day mortality was two (4\%) and primarily was affected by the presence of concomitant injuries; they died due to pneumonia and difficulty weaning from a ventilator. Long-term complications include tracheal stenosis, and phonation problems from injuries to the larynx and RLN were not seen. The tracheostomy was left in three cases. Esophageal repair cases passed well without fistula formation after the 7th postoperative day (POD) and removal of the NGT.

Although complications were registered in $2 \%$ of cases, due to tracheal stenosis, major morbidity is related to infection from long ICU recovery and anastomotic dehiscence with redo.

\section{DISCUSSION}

Cervical tracheal injury is a life-threatening condition. Very rare cases suffering airway trauma is reached to the hospital alive [Prokakis 2014; William 2003]. In our study, the incidence of cervical tracheal injury is $(0.83 \%)$ of total trauma cases and $(0.46 \%)$ of them needed surgical repair.

Mortality in penetrating neck trauma usually is due to an associated vascular injury and is not mandatory due to the airway injury itself [Prokakis 2014]. In our study, one case was associated with severe vascular, esophageal, maxillofacial, and head injuries. The vascular hemorrhage was profuse due to injury of IJV and was very difficult to control after removal of local compression. Emergency exploration was done and controlled by ligation. Another case was associated with esophageal injury and extradural hematoma with severe brain damage that could not be weaned from ventilator until death. Glasgow Coma Scale (GCS) was started at 6 until reaching 3 on the second postoperative day.

The diagnosis requires a high index of suspicion as it is associated with non-specific symptoms and signs. A combination 
Table 1. Demographic features, clinical findings, associated injuries, and postoperative outcome

\begin{tabular}{|c|c|}
\hline Variable & Percentage \% \\
\hline Sex (male) & $49(98 \%)$ \\
\hline Age (years) & $20-40(30 \pm 10)$ \\
\hline \multicolumn{2}{|l|}{ Etiology: } \\
\hline Blunt trauma & $35(70 \%)$ \\
\hline Penetrating trauma & $15(30 \%)$ \\
\hline \multicolumn{2}{|l|}{ Clinical presentation: } \\
\hline Subcutaneous emphysema & $49(99 \%)$ \\
\hline Hemoptysis & $15(30 \%)$ \\
\hline Neck contusions & $15(30 \%)$ \\
\hline Respiratory distress & $1(2 \%)$ \\
\hline Open neck wound & $1(2 \%)$ \\
\hline \multicolumn{2}{|l|}{ Associated injuries: } \\
\hline Esophageal perforation & $4(8 \%)$ \\
\hline Vascular injuries & $4(8 \%)$ \\
\hline Head injury & $1(2 \%)$ \\
\hline Maxillofacial injuries & $3(6 \%)$ \\
\hline \multicolumn{2}{|l|}{ Endotracheal intubation: } \\
\hline Easy intubation & $46(92 \%)$ \\
\hline Tracheostomy & $3(6 \%)$ \\
\hline Distal end of transected trachea & $1(2 \%)$ \\
\hline Mean hospital stay (days) & 9.5 days (range, $7-14$ days) \\
\hline \multicolumn{2}{|l|}{ Postoperative complications: } \\
\hline Pneumonia & $4(8 \%)$ \\
\hline Septic shock & $2(4 \%)$ \\
\hline Prolonged ventilation ( $>10$ days) & $1(2 \%)$ \\
\hline Mortality & $2(4 \%)$ \\
\hline
\end{tabular}

of MDCT and bronchoscopy is the gold standard to determine the site, extent, and even small tear of tracheal injury [Zhao 2017; Yasser 2018]. MDCT and/or bronchoscopy were used in our study.

Air escape from a penetrating neck trauma should be considered diagnostic for airway injury [Prokakis 2014]. In our study, there were five cases with air escape from a neck wound, $33 \%$ from a sharp neck injury and $10 \%$ of total cases.

Morbidity after repair includes infection, anastomotic dehiscence, stenosis, and phonation problems [Yasser 2018]. In our study, there was only one case of tracheal stenosis.

RLN injury previously was reported in about $60 \%$ of cases with complete cervical trachea transection [Yasser 2018]. We did not record any case of RLN injury in our study.

The tragedy of cervical tracheal injury is that (78\%) of the victims are men in the adult age (20-44 years old), causing significant impact on productivity, if not treated well [Prokakis 2014]. In our study, almost all cases were adult male (98\%) (age range, 20-40). So, emergency tracheal repair after early diagnosis is very important to improve quality of life.

In penetrating neck injuries associated with cervical tracheal trauma, rupture of the esophagus is the most common associated injury occurring in $43 \%$ of cases [Prokakis 2014]. In our study, the incidence of esophageal injury was $8 \%$ of sharp trauma associated with cervical trachea injury.

Time management is very important. The presence of an interdisciplinary team with high experience of anesthesia is mandatory to ensure high quality management with a low morbidity and mortality rate [Beshay 2020].

\section{CONCLUSIONS}

The outcome of emergency cervical tracheal repair after non-iatrogenic trauma is satisfactory. A high index of suspicion is needed because there is no specific clinical presentation. MDCT and/or bronchoscopy (rigid or FOB) are mandatory in stable patients for early diagnosis.

\section{REFERENCES}

Beshay M, Mertzlufft F, Kottkamp H, et al. 2020. Analysis of risk factors in the thoracic trauma patients with a comparison of a modern trauma center: a mono-center study. WJ Emerg Surg. 15:45- 55.

Cheng-Yu C, Shih-Lung C, Shih-Chieh C. 2011. Conservative Treatment of Severe Tracheal Laceration after Endotracheal Intubation. Respir Care.56(6):861-62.

Eduardo M, Javier B, Maria A, Javier L, Pedro M, Alejandro G. 2009. Tracheal rupture after endotracheal intubation: a literature systematic review. Euro J Cardiothorac Surg. 35: 1056-62.

Prokakis C, Efstratios N, Panagiotis D, et al. 2014. Airway trauma: a review on epidemiology, mechanisms of injury, diagnosis and treatment. J Cardiothorac Surg. 9:117-25.

Shweel M, Shaban Y. 2013. Radiological evaluation of post-traumatic tracheal stenosis using multidetector CT with multiplanar reformatted imaging and virtual bronchoscopy: Comparison with intraoperative findings. Egypt J Radiol Nucl Med. 44: 513-21.

Weil P, Dong Y, Jiapeng H, et al. 2019. Anesthetic management of tracheal laceration from traumatic dislocation of the first rib: a case report and literature of the review. BMC Anesthesiol. 19:149-55.

William E, Ruben P. 2003. Management of tracheal trauma. Can J Anesth. 50 (6); $1-6$.

Yasser A, Ahmed A. 2018. Blunt Complete Tracheal Transection. Indian J Surg.

Yasser A, Ahmed A. 2018. Challenges in the Initial Management of Complete Tracheal Transection after Blunt Neck Trauma. Blunt Complete Transection of Trachea. JOJ Case Stud. 6(3): 555687.

Yasser A, Ahmed O, Mostafa K, Mostafa S. 2018. Rare case of survivor after accidental hanging and complete tracheal transection. Case report. Am Res J Clinic Case Reports. 1(2); 1-9.

Zhao Z,Tianyi Z, Xunliang Y, Jinbo Z, Xiaofei L, Yongan Z. 2017. Update on diagnosis and treatment of tracheal and bronchial injury. J Thorac Dis. 1(9):50-56. 